\title{
Performance Analysis of a Manipulation Task in Time-Delayed Teleoperation
}

\author{
Michael C. Yip, Mahdi Tavakoli, Member, IEEE, and Robert D. Howe, Senior Member, IEEE
}

\begin{abstract}
Abtract-There is ample research on the stabilization of haptic teleoperation systems under communication time delay. Little attention, however, has so far been paid to the usefulness of delayed haptic feedback on task performance. While the usefulness of haptic feedback in no-delay teleoperation has been previously established, this paper investigates whether haptic feedback helps to improve task performance in the presence of delay. We consider peg-in-the-hole insertion, which is a dexterous manipulation task requiring high force levels at certain points during the task execution. Through a user study involving unilateral and bilateral teleoperation experiments under different delays, it is observed that in both unilateral and bilateral teleoperation, the task completion time increases as delay increases. It is also seen that haptic feedback helps reduce the amount and rate of energy transfer to the environment and the occurrence of larger robot/environment interaction forces. However, with the users mindful of minimizing contact forces, haptic feedback causes the task to take more time compared to no haptic feedback regardless of the time delay. Thus, for tasks where low completion times are crucial given a tolerance for larger forces, unilateral feedback may be sufficient.
\end{abstract}

\section{INTRODUCTION}

As a human operator controls the position of a teleoperated robot through a user interface and performs a task such as grasping an object in the remote environment, capturing the robot-object contact forces and bringing them to the operator in the form of haptic feedback will engage the operator's sense of touch and may enable better control over the contact forces. The main goals of such a haptic teleoperation system are (1) stability and (2) transparency which is defined as the ability to present undistorted dynamics of the remote environment to the human operator [1]. An interesting control problem arises from the presence of a non-negligible time delay in the communication media between the user interface (master) and the teleoperated robot (slave), which severely affects the stability and transparency of the haptic teleoperation system. Several approaches have been proposed in the literature to deal with this problem [2-5].

The passivity-based approach to delay compensation, which has been expressed in the scattering framework [6] and in the wave transformation framework [7], is an efficient method for stabilizing a teleoperation system independent of transmission delays. The key to this approach is making the non-passive delayed communication medium passive (at the expense of transparency degradation). This approach does not eliminate or reduce the delay, thus the potentially harmful effects of delay on performance is persisting.

While there is ample research on delayed teleoperation system stabilization, little attention has been paid to the effect of delayed haptic feedback on task performance - i.e., whether, and for what class of tasks, haptic interaction actually improves task performance in delayed teleoperation. While the usefulness of haptic feedback in no-delay

M.C. Yip is with the Dept. of Elec. and Comp. Eng., University of British Columbia, Vancouver, BC V6T1Z4, CAN (e-mail: myip@ece.ubc.ca).

M. Tavakoli is with the Dept. of Elec. And Comp. Eng., University of

Alberta, Edmonton, AB T6G2V4, CAN (e-mail: tavakoli@ece.ualberta.ca).

R. D. Howe is with the Harvard School of Eng. and App. Sci., Harvard

University, Cambridge, MA 02138 USA (e-mail: howe@ seas.harvard.edu). teleoperation has been established, e.g., [8-10], the loss of temporal coincidence between the operator's motions and the ensuing reflected forces in delayed teleoperation may confuse the operator so much so that the force feedback becomes useless or even misleading. Thus, this paper aims to study how delayed haptics affects the user task performance in a typical manipulation task.

To assess the value of providing haptic feedback to the user during delayed teleoperation, prior work studied the effect of delay on the human's perception of the relative stiffness of virtual spring-like surfaces simulated by reflecting forces proportional to the user's virtual surface indentations. Subjected to a forced-choice paradigm (i.e., distinguish the stiffer of two surfaces or identify them as having the same stiffness), users perceived the surfaces to be stiffer than actual under delayed force feedback and the stiffness overestimation increased for larger delays [11,12]. Recently, the effect of crossing the boundary of a force field, where local stiffness is ill-defined, on the perception of delayed stiffness has been studied [13]. It was found that subjects interacting with delayed force fields underestimate (overestimate) stiffness if they do not move (do move) across the boundary of the elastic field.

While the literature has so far been limited to studying the stiffness discrimination task, we consider the effect of delayed haptic feedback on the performance of a peg-in-thehole insertion task. The differences are in that stiffness discrimination is a single-DOF, low-force sensing task while peg-in-the-hole insertion is a multi-DOF, high-force manipulation task. The complexities of this dexterous manipulation task are likely to affect the usefulness of haptic information in the presence of delay.

It is noteworthy that haptic feedback has been shown to help task performance in no-delay teleoperation in different ways depending on the levels of forces [10]. At high levels of force feedback, environment mechanical properties are presented against the user's hand as passive physical constraints, which serve both as safety barriers and as intuitive guides for tools. At low levels of force feedback, however, force feedback is beneficial less as a physical constraint and more as a supplemental information source requiring an increased level of awareness and cognitive processing by the user. Despite the fact that this study was performed under no delay, the existing difference in the underlying mechanisms of haptic assistance motivated us to consider the peg-in-the-hole insertion task, which requires the user to exert high forces at some points during the operation and to accurately coordinate the insertion forces in multiple directions for the entire duration of the task.

A general hypothesis supported by past work [11-13] is that time delays in position feedforward and haptic feedback can disturb the human operator and cause misjudgements. The key question we pose in this paper is how is the user performance of this basic manipulation task influenced by delays in haptic feedback? This work should not be confused with prior work analytically or empirically studying how wave transformations limit the performance of teleoperation systems due to displaying distorted inertia and damping to the operator, the wave reflection phenomenon, etc. Instead of system performance, we directly study task performance 
(user performance) under delayed haptic feedback through teleoperation experiments done by a pool of human subjects.

The main contribution of the paper is studying the combined effects of time delay and feedback modality on task performance. Past user studies have looked at either the effect of haptic feedback on task performance in the absence of delay, or at the effect of various delays on task performance in the absence of haptic feedback (i.e., onedimensional comparisons). In this paper, however, our twodimensional comparison of task performance is carried out across both various modalities (haptic feedback and no haptic feedback) and various delays.

This paper is organized as follows. In Section 2, we describe the experimental setup including the teleoperation system and how it is stabilized under time delay. Section 3 describes the experiments protocole when users executed a peg insertion task under various delays and explains how the task was segmented and evaluated in terms of performance. Section 4 presents the results such as the effect of delay on task completion time and the effect of feedback modality on the energy transfer to the environment. Section 5 discusses the results, and Section 6 includes concluding remarks.

\section{EXPERIMENTAL SETUP}

Two PHANToM Premium 1.5 devices (SensAble Technologies, Inc., Woburn, MA, USA) were used, one as the master device and one as the slave device. Figure 1 shows the experimental setup. On the master side, the user manipulates the first PHANToM robot and the motion commands are transmitted to the slave side through a delayed communication channel. On the slave side, an aluminum cylindrical peg is attached to the endpoint of the second PHANToM robot, which is placed next to an aluminum block with a cylindrical hole slightly larger than the peg. The interaction forces between the peg (i.e., the slave robot endeffector) and the hole are transmitted back to the master side through a similar delayed communication channel. A standard video camera is aimed 45 degrees downwards at the slave-side environment (i.e., the peg and the hole), capturing a video feed of the workspace and streaming it to the operator. The video feed is designed to suffer from the same delays as the motion commands and the force feedback.

Peg-in-the-hole setups are generally characterized using a precision value

$$
I=\log _{2}\left(d_{H} /\left(d_{H}-d_{P}\right)\right)
$$

where $d_{H}$ is the diameter of the hole and $d_{P}$ is the diameter of the peg. The parameters of our peg-in-the-hole setup are $d_{H}=$ $18.38(\mathrm{~mm}), d_{P}=18.60(\mathrm{~mm})$, peg height $h=52(\mathrm{~mm})$, and hole tilt angle $\theta=21(\mathrm{deg})$. The precision value of our experiment is $I=1.922$. Other studies used precision values within the same order of magnitude [14].

(a)
We used the passivity-based wave variable method [7] for stabilizing the master-slave system under time delay. A pair of wave variables $(u, v)$ is defined based on a pair of standard power variables, i.e., velocity and force $(V, F)$, by

$$
u=(b V+F) / \sqrt{2 b}, \quad v=(b V-F) / \sqrt{2 b}
$$

The characteristic wave impedance $b$ is a positive constant and must be tuned in order to trade off the speed of motion for the levels of force feedback [7]. In general, when the wave impedance is decreased (increased), force levels are lower (higher) and the system shows less (more) damping to the operator. In the experimental setup, we tuned the wave impedance value through trial and errors aimed achieving both easy motion (low damping) when the slave is in free space and high levels of force feedback when the slave is in contact with an environment.

Interestingly, using a pair of such wave transforms at the two ends of a delayed communication channel can make it passive. Such a "passivated" (delay-compensated) channel guarantees the stability assuming the remote environment and the operator display passive behaviors.

In this paper, the communication delay $T_{d}$ has been assumed to be constant and equal in both directions (even though the assumption of equal forward and backward delays is not necessary for ensuring passivity). The resulting control architecture with the delay-compensated channel and using the position error-based (PEB) controller is shown in Figure 2. As can be seen, the PEB controller does not use any force sensor measurements and merely tries to minimize the difference between the (delayed) master position/velocity and the slave position/velocity, thus reflecting a force to the user once the slave makes contact with an object.

\section{PROCEDURE}

There are two study variables that characterize our peg-inthe-hole experiments: teleoperation modality (unilateral or without haptic feedback; bilateral or with haptic feedback), and delay $(0 ; 100 ; 200 ; 500 \mathrm{~ms})$ - this spans the range typically encountered in current teleoperation systems. The delay values are for unidirectional communication, and therefore, round-trip signals experience double the delay time. The video stream from the slave side to the master side lags by the delay amount, too.

Eight adult subjects (six male and two female; average age of 28) with little past exposures to haptic feedback participated in the experiments. Peg-in-the-hole insertion under each modality/delay pairing (i.e., eight different experimental conditions) was performed twice by each subject. Therefore, the total number of trials was $N=128$ (sixteen trials for each experimental condition). The order in which modality/delay pairings were presented to a subject was randomized in order to minimize the effect of learning.

(b)

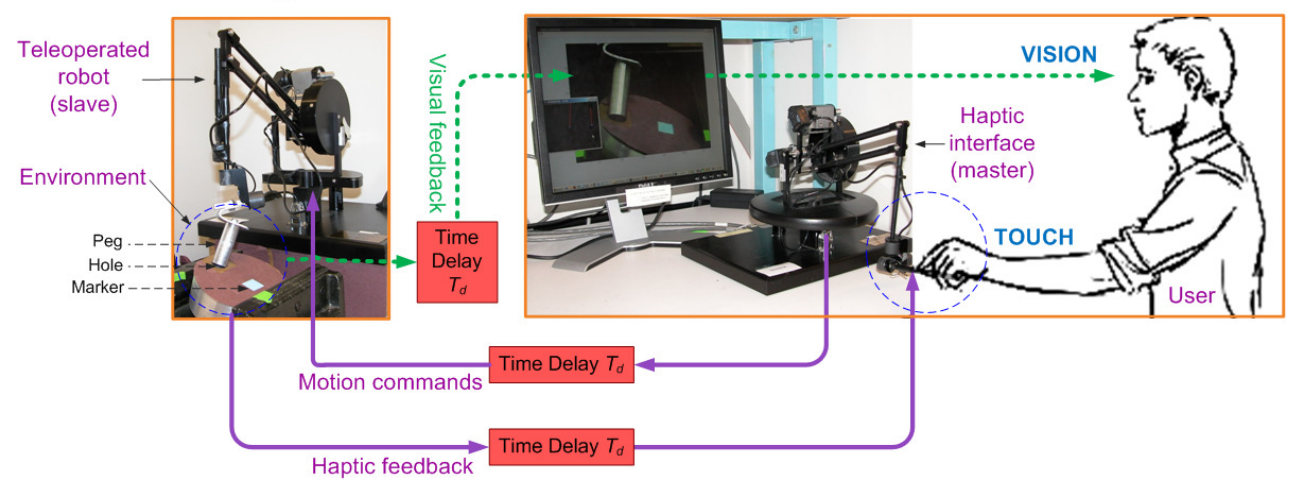

Figure 1. The teleoperated robot and the peg/hole setup (a), and the user interface (b). 
Before the experiments, subjects were given a few practice trials (covering the entire range of delays for both feedback modalities) to familiarize themselves with the operation of the master-slave system and the execution of the task. In each trial, the master and slave positions and the task completion time were recorded, and forces in 3D space were computed. The subjects were asked at the beginning of experiments to minimize the levels of contact forces as well as the task completion times.

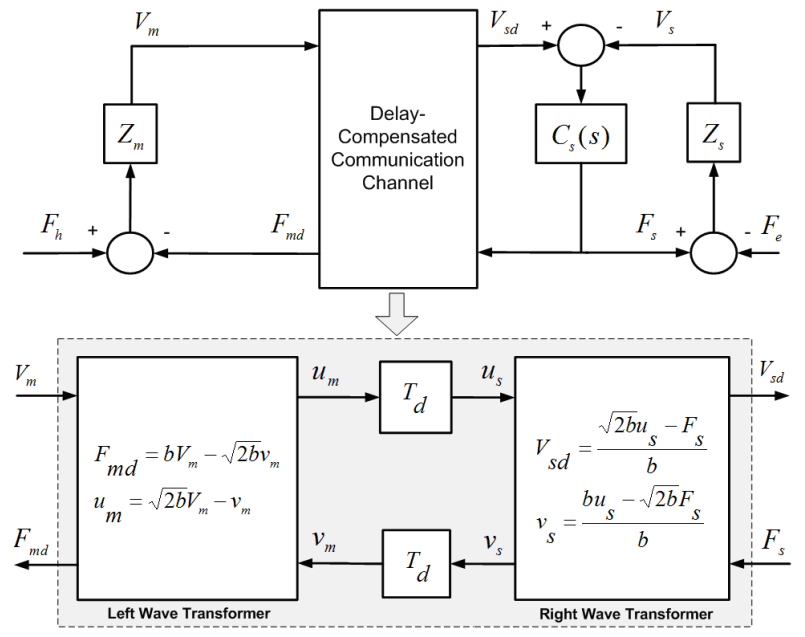

Figure 2. Passivity-based, delay-compensated PEB teleoperation system.

\section{A. Task Sequence and Segmentation}

A peg-in-the-hole run started when the peg was inside the hole and consisted of the following four subtasks:

1. Retract the peg from the hole,

2. Move to a distant position, tap a marker, return the peg to the hole entrance,

3. Align the peg with the hole, and

4. Insert the peg into the hole.

The distant position, about $40 \mathrm{~mm}$ from the hole, was used to separate peg retraction from peg alignment. After the eight participants completed the experiments and all data was gathered, data segmentation was performed by determining the radial distances of the peg from three points: a start location (determined when the peg is fully inside the hole), a far-off location (determined when the peg touches a marked position far from the hole), and an alignment location (determined when the peg is aligned prior to insertion). When the trajectory of the peg leaves one point's radial vicinity, it enters a new subtask. Out of the four subtasks listed above, we were only concerned about the retraction, alignment, and insertion subtasks. The segmentation of a sample trajectory is shown in Figure 3, showing the alignment effort of the operator.

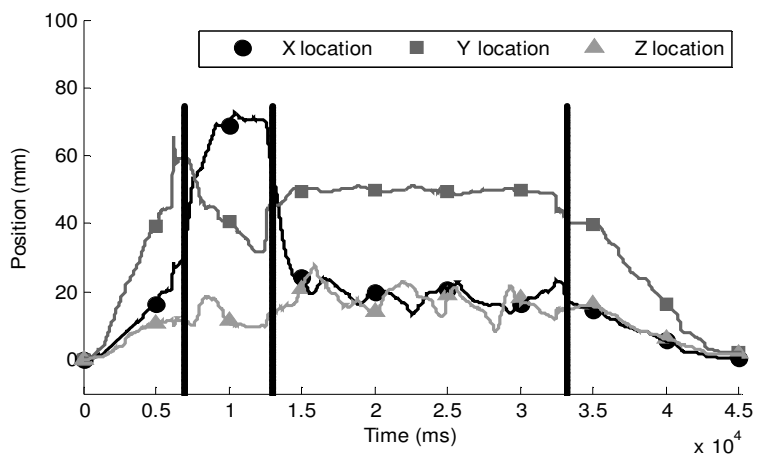

Figure 3. Segmentation of a 3D trajectory (shown in three dimensions).

\section{B. Performance Measures}

We considered the following metrics of performance of the task under different experimental conditions:

Completion Time: Times were saved for subtasks (extraction/alignment/insertion) and the entire task.

Sum of Squared Forces and Average Sum of Squared Forces: The sum of squared forces (SOSF) is used to give an absolute measure of the force interactions between the slave and the environment. We are also interested in the average sum of squared forces as it gives an indication of the rate at which forces are being applied to the environment. If $|F|$ is the absolute force magnitude and $n$ is the number of data samples, we have:

$$
\begin{aligned}
& S O S F=\sum_{i=1}^{n}|F|^{2} \\
& A S O S F=\frac{1}{n} \sum_{i=1}^{n}|F|^{2}
\end{aligned}
$$

Both SOSF and ASOSF can also show the amount and rate of effort required by the operator to perform the task.

Force Histograms: Force histograms show the frequency of occurrence of different force levels, and are effective in showing how forces and completion times interact.

\section{RESULTS}

Figure 4 shows the mean time required to complete each subtask under different delays. Regardless of the teleoperation modality, the task completion times for all subtasks increase as delay increases, with overall task completion times increasing from $14.3 \mathrm{~s}$ to $34.1 \mathrm{~s}$ (unilateral) and from $18.9 \mathrm{~s}$ to $39.7 \mathrm{~s}$ (bilateral) as the delay increases from 0 to $500 \mathrm{~ms}$. When the users are given haptic feedback, the completion times increase by about $5 \mathrm{~s}$ compared to the unilateral mode for all delays levels. This is due to the fact that, during the insertion and extraction phases, force feedback increases the times by approximately 2 to $3 \mathrm{~s}$ at each delay level. In order to determine the statistical significance of the results, a two-tailed student $t$-test was used to obtain a probability that the null hypothesis is true $(t$ test for retraction: $\mathrm{p}_{0}=0.00098, \mathrm{p}_{100}=0.0012, \mathrm{p}_{200}=0.0023$, $\mathrm{p}_{500}=0.042 ; t$-test for insertion: $\mathrm{p}_{0}=0.052, \mathrm{p}_{100}=0.076, \mathrm{p}_{200}$ $\left.=0.030, \mathrm{p}_{500}=0.00011\right)$. In contrast, alignment times (Figure 4b) do not show a significant difference between unilateral and bilateral control for all delays ( $\mathrm{t}$-test for alignment: $\mathrm{p}_{0}=$ $\left.0.62, \mathrm{p}_{100}=0.71, \mathrm{p}_{200}=0.65, \mathrm{p}_{500}=0.36\right)$, so haptic feedback or the lack of it does not significantly affect the time required to align the peg with the hole.

Figure 5 shows the task completion time and its standard error at each delay across the two teleoperation modalities. Note that Figures 5a-d essentially display the same results as in Figure 4 but in a different way.

The standard errors in Figure 5 have been normalized with the mean completion time of the specific subtask. We can see that during alignment (Figure 5f) and regardless of teleoperation modality, the standard errors decrease as the delay increases, indicating that the time required to complete the alignment subtask becomes less variable across different subjects. This means that the users are able to perform the alignment task more consistently than the other subtasks. This trend is not apparent for retraction and insertion subtasks (Figure $5 \mathrm{e}$ and $5 \mathrm{~g}$ ).

Figure 6a-d shows the the sum of squared forces (SOSF) at each delay. To compare these results, we performed an analysis of variance (ANOVA) to determine the statistical significance across delays. Results show that the presence of haptic feedback reduces the SOSF at each delay. In addition, it effectively stabilizes the SOSF from changing significantly as the delay increases (ANOVA across delays: $F=1.3028, p$ $=0.2834)$. In comparison, unilateral feedback does show 
significant increases in sum of squared forces as the delay increases (ANOVA across delays: $F=3.5462, p=0.0206$ ).

We normalize the sum of squared forces by time in order to obtain an average sum of squared forces (ASOSF) value. Figure 7a-d show that unilateral teleoperation causes more forces on average to be exerted on the environment compared to bilateral teleoperation. Bilateral teleoperation maintains a nearly constant ASOSF across delays (ANOVA across delays: $F=0.6461, p=0.5889$ ). However, contrary to the delay-dependant SOSF for unilateral teleoperation, the ASOSF for unilateral teleoperation does not increase as significantly across delays (ANOVA across delays: $F=$ 0.4037, $p=0.7509$ ). It was also observed (although not shown here) that the standard errors for unilateral teleoperation were generally higher than for bilateral teleoperation, indicating that the variance in the ASOSF is higher when the subject is only provided unilateral feedback. Figure 8 shows the force histogram distributions for all delays. During alignment (Figure 8b), the addition of haptic feedback does not significantly change the force distribution. However, bilateral teleoperation results in fewer occurrences of high force values across all delays during retraction and insertion. Unilateral feedback induces large forces more often but also fewer small forces due to its faster completion time. For retraction and insertion, while the general shapes and peaks of unilateral force distributions do not significantly change as the delay increases, the peaks of bilateral force distributions show an increase in occurrence as the delay increases (Figure 8a,c).
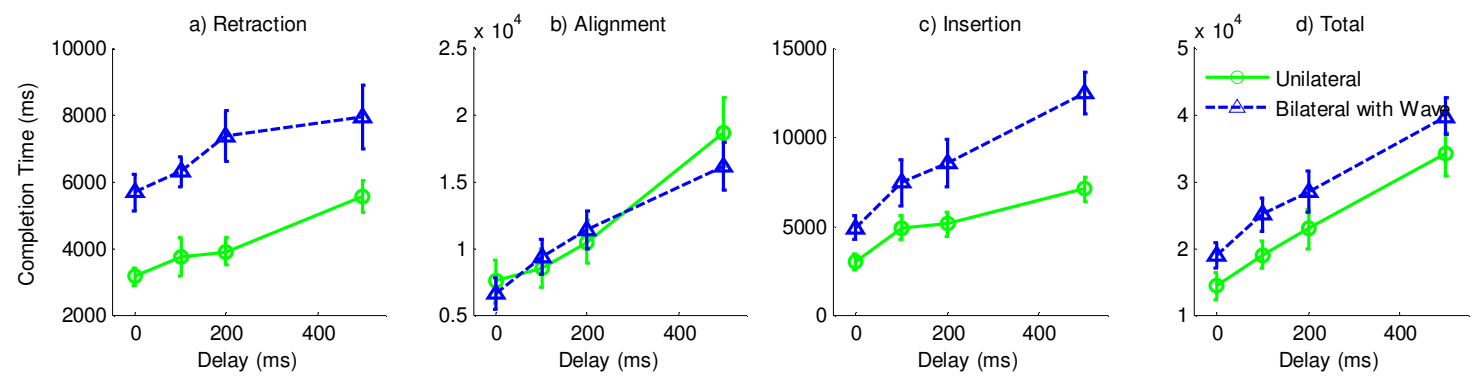

Figure 4. Task completion times across delay. Bars show standard error
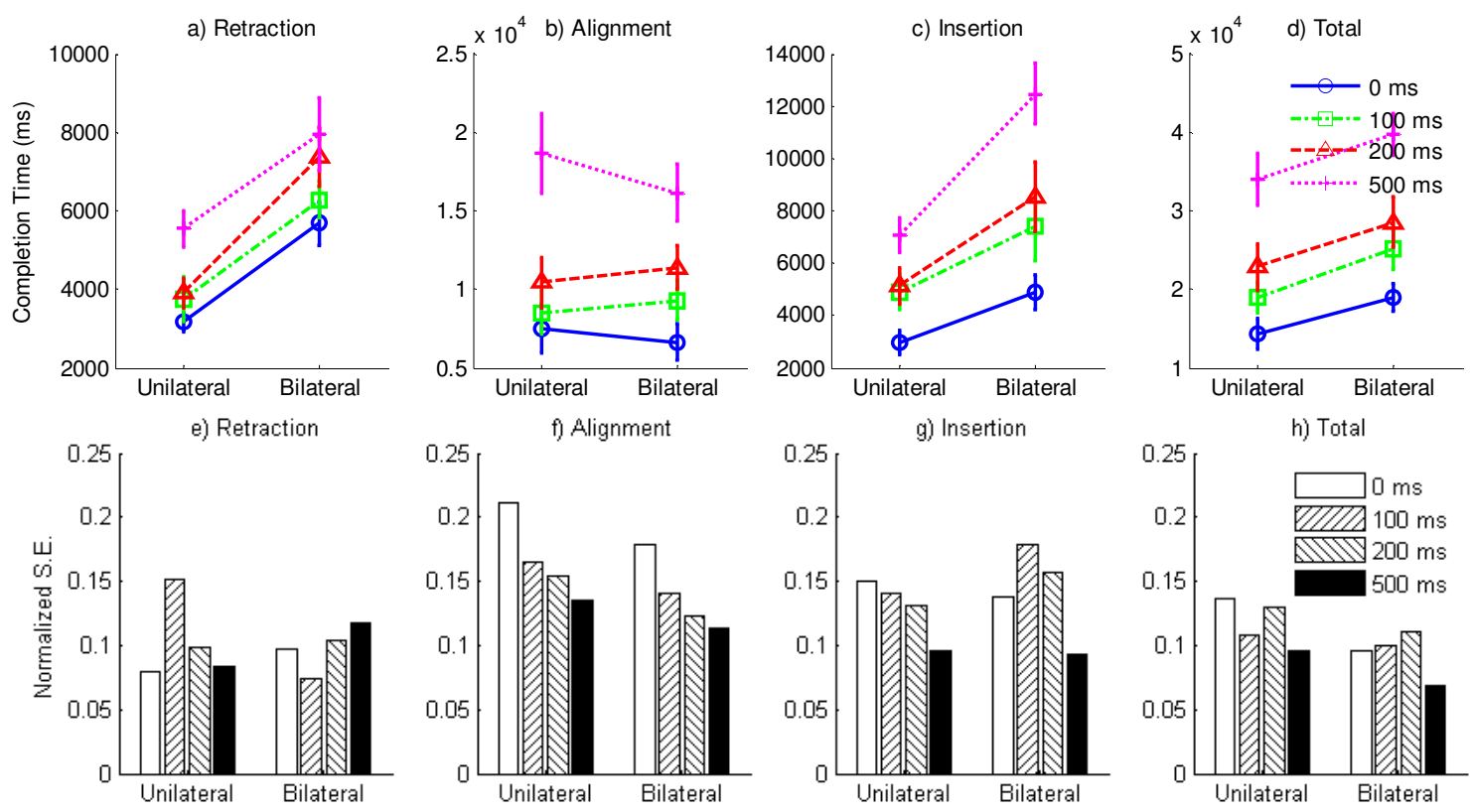

Figure 5. Task completion times and corresponding normalized standard errors across modality.
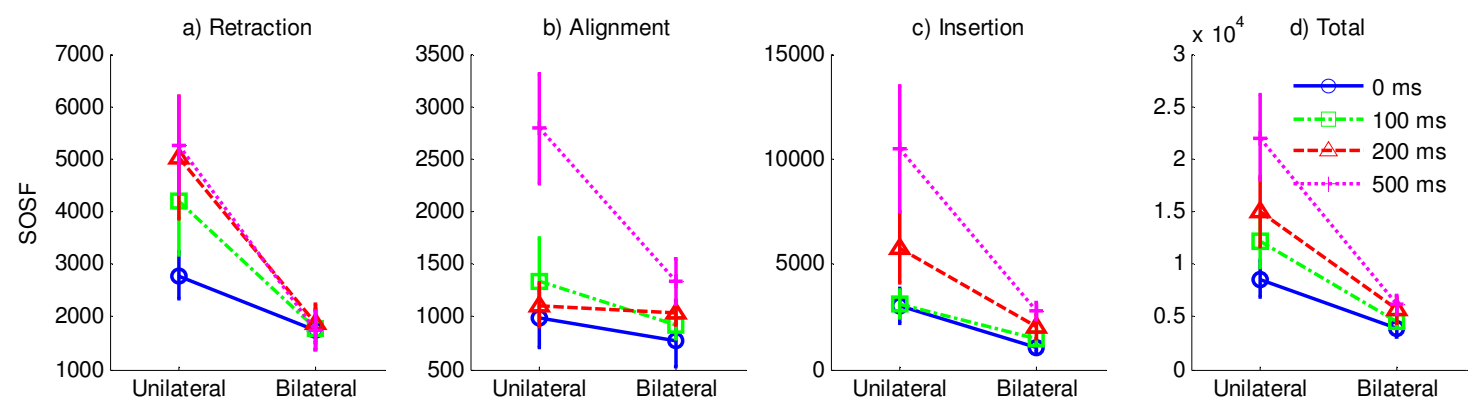

Figure 6. Sum of squared forces (SOSF) across modality. 

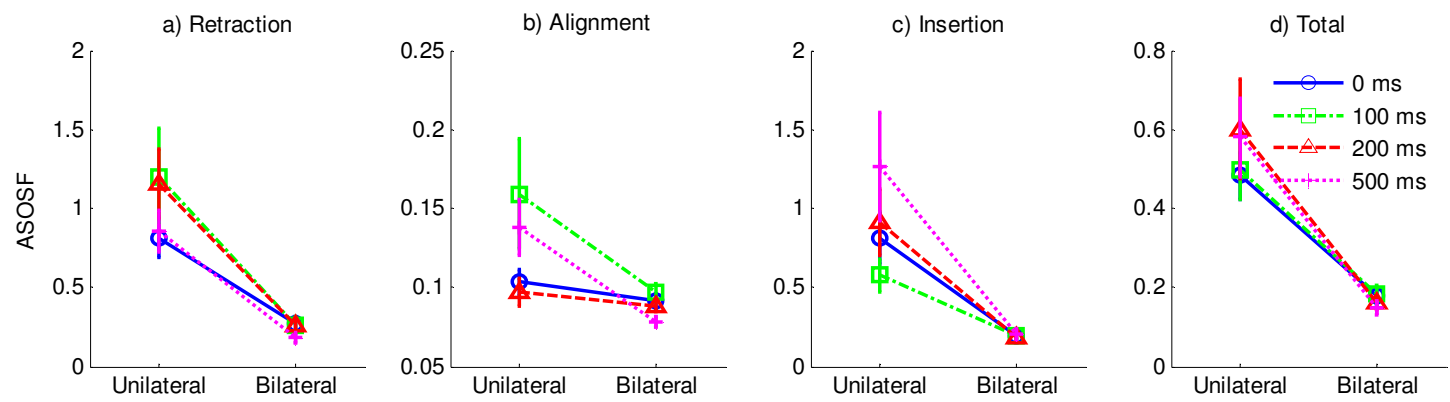

Figure 7. Average sum of squared forces (ASOSF) across modality.
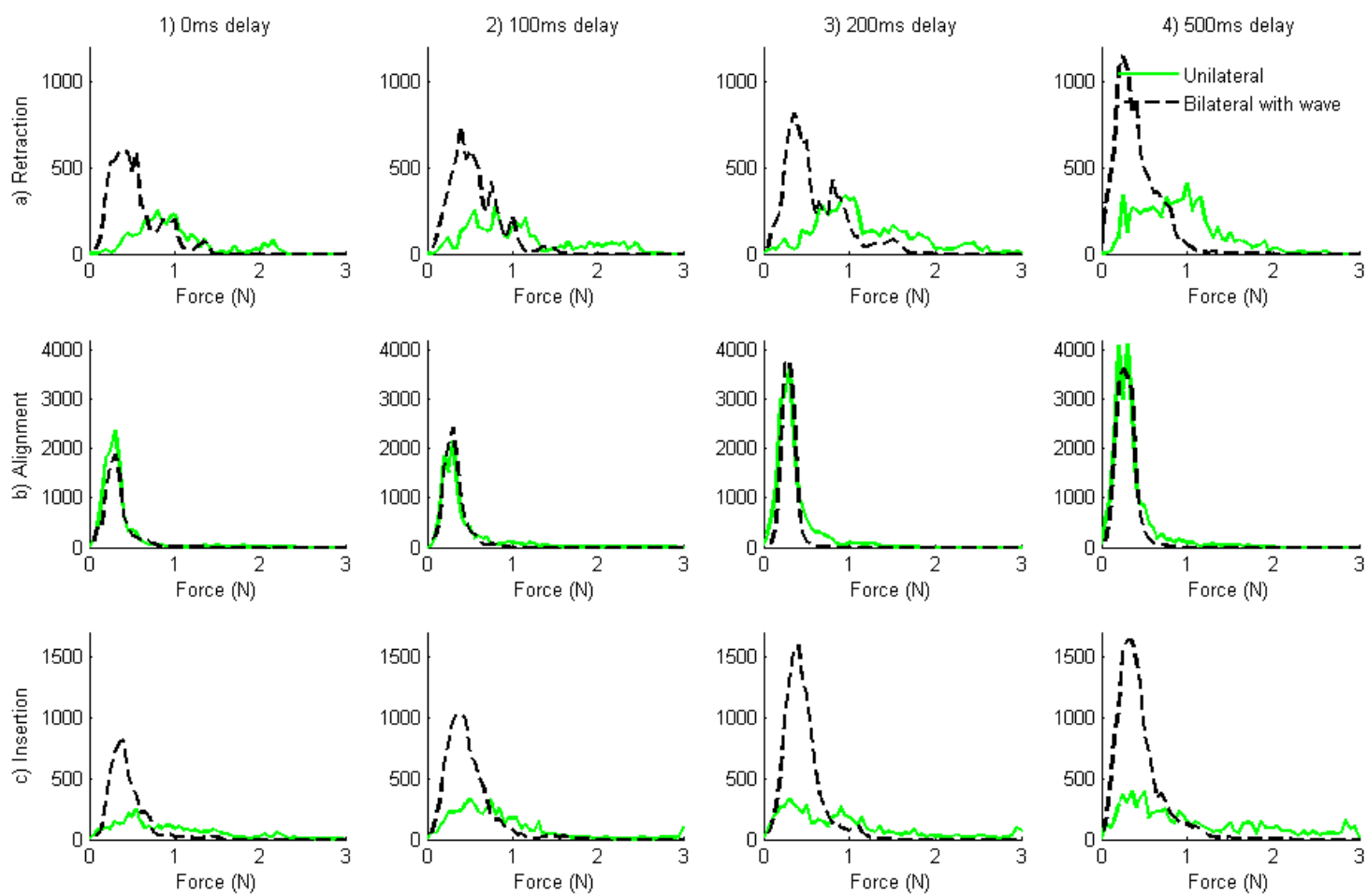

Figure 8. Force histogram distribution across delay.

Table 1. Summary of the effects of unilateral and bilateral control on task performance metrics (under delay)

\begin{tabular}{ccc}
\hline \hline Metric $\backslash$ Modality & Unilateral & Bilateral \\
\hline $\begin{array}{c}\text { Time required to } \\
\text { complete the task }\end{array}$ & less time & more time \\
$\begin{array}{c}\text { Force interactions } \\
\text { (SOSF, ASOSF) }\end{array}$ & higher forces & lower forces \\
Force Histograms & $\begin{array}{c}\text { forces lie over a } \\
\text { larger range }\end{array}$ & $\begin{array}{c}\text { forces lie over a } \\
\text { tighter range }\end{array}$ \\
\hline \hline
\end{tabular}

\section{DISCUSSION}

Studies have shown that teleoperation with haptic feedback allows users to perform certain tasks with greater success than without haptic feedback. However, when teleoperation is performed under a significant delay, the fidelity of haptic teleoperation is limited. In this paper, we directly measured the performance benefits of delayed force feedback in a simple assembly task. This was accomplished by comparing bilateral teleoperation to unilateral teleoperation across different delays, and characterizing performance based upon the time required to complete a task and the forces exerted against the environment. Summarized versions of the results are given in Table 1.

The results show that bilateral teleoperation causes tasks to take more time compared to unilateral teleoperation regardless of the time delay. The difference was observed only during the insertion and extraction phases of the task, which is consistent with the observation that good coordination of forces is needed to accomplish closetolerance assembly tasks [15], and that the alignment subtask is largely position constrained. This result suggests that, given haptic feedback, users are more mindful of minimizing forces, which comes at the expense of speed.

We also showed that regardless of feedback modalities, completion times still increase significantly as delay increases, and therefore bilateral teleoperation was shown to take the longest time across all delays. For teleoperation applications where the completion time is the highest priority constraint, these results suggest that not providing additional feedback may in fact be a better option than providing haptic feedback as the user will likely require more time to complete a task when given feedback from these modalities. 
Although haptic feedback increases the completion time of all tasks, we saw that it reduces the applied force regardless of the time delay. In particular, the sum of squared force and average sum of squared force evaluations indicated that both indicated that bilateral feedback could consistently maintain low forces with low variability even across increasing delays. Therefore, haptic feedback helps for tasks where minimizing the amount of forces applied to objects and the environment is critical. In addition, we showed that haptic feedback helps minimize the occurrences of larger forces and subjects the task environment to lower forces. However, since the tasks take more time to complete, the lower forces are applied on the environment for a longer period of time. This inverse relationship between time and force minimization is an apparent tradeoff when haptic feedback is used in teleoperation systems under delay. Nevertheless, the introduction of haptic feedback still minimizes the total number of occurrences of large forces when compared to unilateral feedback, as shown in the force distributions. Therefore, in situations where minimizing the maximum force seen by the environment is a primary concern, haptic feedback becomes beneficial.

We note that this initial study is specific to a particular type of task, i.e. assembly and disassembly of close-fitting parts. While this is perhaps in the category of tasks where force reflection is most clearly beneficial, further study will be required to understand the benefits of haptic feedback in general. The master and slave devices used in the experiments have relatively low stiffness and inertia compared to industrial manipulators. For manipulators with a higher stiffness or inertia, we speculate that the transparency of the system may be different the task performance would change accordingly.

In addition, this study used the wave variable approach for ensuring stability (and passivity) under time delay. Wave variables were chosen due to their popularity as robust stabilizers for delayed teleoperation systems and the well-characterized wave-based system performance. Wary of the adverse effect of wave transformations on system performance, however, it is interesting to speculate that alternative approaches to time delay compensation in delayed teleoperation might result in better task performance, if it is possible to identify the aspects of force feedback that are most important to preserve under delay. On the other hand, if the advantage in terms of system performance offered by any other delay compensation method over the wave-based method that we have used is incremental, the adverse effects of delay on task performance are expected to persist, yielding results similar to those reported in this paper.

The delay range considered here (up to $500 \mathrm{~ms}$ ) is representative of most applications in terrestrial and nearearth environments; e.g., the round-trip time for communication via geostationary satellites is approximately half a second. Larger delays may be encountered in interplanetary space missions, and larger and variable delays are potentially relevant for teleoperation over the internet. Again, further study will be required to address these situations.

\section{CONCLUSION}

In this paper, we considered the usefulness of haptic feedback for task performance in the presence of delay. We evaluated a peg-in-the-hole insertion task through a user study involving unilateral and bilateral teleoperation experiments under different delays. We found that

1. When the users are asked to minimize applied force, bilateral teleoperation causes the task to take more time compared to unilateral teleoperation, regardless of time delay.

2. The completion time increases as the delay increases, regardless of teleoperation modality.

3. The presence of haptic feedback reduces the applied force, and the applied force does not depend on the magnitude of the time delay.

4. Haptic feedback helps minimize the occurrences of high forces.

These results suggest that, in a delayed system, the addition of haptic feedback is beneficial in situations where minimizing the maximum force seen by the environment is the key constraint. Otherwise, when one is not interested in lowering the robot/environment contact forces, unilateral teleoperation is sufficient and even results in faster completion times.

\section{REFERENCES}

[1] B. Hannaford, "A design framework for teleoperators with kinesthetic feedback," IEEE Transactions on Robotics and Automation, vol. 5, no. 4, pp. 426-434, 1989.

[2] P. F. Hokayem and M.W. Spong, "Bilateral teleoperation: An historical survey," Automatica, vol. 42, no. 12, pp. 2035-2057, 2006.

[3] P. Arcara and C. Melchiorri, "Control schemes for teleoperation with time delay: A comparative study," Robotics and Autonomous Systems, vol. 38, pp. 49-64, 2002.

[4] S. Mascolo, "Modeling the Internet congestion control using a Smith controller with input shaping," Control Engineering Practice, vol. 14, no. 4, pp. 425-435, 2006.

[5] C.L. Lin, C. H. Chen and H. C. Huang, "Stabilizing control of networks with uncertain time varying communication delays," Control Engineering Practice, vol. 16, no. 1, pp. 56-66, 2008.

[6] R.J. Anderson and M.W. Spong "Bilateral control of teleoperators with time delay," IEEE Transactions on Automatic Control, vol. 34, no. 5, pp. 494-501, 1989.

[7] G. Niemeyer and J. J. E. Slotine, "Stable adaptive teleoperation," IEEE Journal of Oceanic Engineering, vol. 16, no. 1, pp. 152-162, 1991.

[8] B. Hannaford and L. Wood, "Performance evaluation of a 6 axis high fidelity generalized force reflecting teleoperator," The JPL/NASA Conf. Space Telerobotics, 1989, pp. 89-97.

[9] G. C. Burdea, Force and touch feedback for virtual reality, New York: Wiley, 1996.

[10] C. Wagner and R. Howe, "Force feedback benefit depends on experience in multiple degree of freedom robotic surgery task," IEEE Trans. Robotics, vol. 23, no. 6, pp. 1235-1240, 2007.

[11] A. Pressman, L.J. Welty, A. Karniel, F.A. Mussa-Ivaldi, "Perception of Delayed Stiffness", Int. J. Robotics Research, vol. 26, no. 11-12, pp. 1191-1203, 2007.

[12] H. Ohnishi and K. Mochizuki, "Effect of Delay of Feedback Force on Perception of Elastic Force: A Psychophysical Approach," IEICE Trans. Communications, vol. E90-B, no. 1, pp. 12-20, 2007.

[13] I. Nisky, F. A. Mussa-Ivaldi and A. Karniel, "A Regression and Boundary-Crossing-Based Model for the Perception of Delayed Stiffness," IEEE Trans. Haptics, vol. 1, no. 2, pp. 73-83, 2008.

[14] B. Hannaford, L. Wood, D.A. McAffee, H. Zak, "Performance Evaluation of a Six-Axis Generalized Force-Reflecting Teleoperator", IEEE Trans. on Systems, Man. And Cybernetics, vol. 21, no. 3, pp. 620-633, Jun. 1991.

[15] D.E. Whitney, "Quasi-Static Assembly of Compliantly Supported Rigid Parts," Journal of Dynamics System, Measurement, and Control, vol. 104, pp. 65-77, 1982. 